\section{Retinal pigment epithelial tear following photodynamic therapy for choroidal neovascularization
secondary to AMD}

\author{
Abstract \\ Purpose To describe retinal pigment \\ epithelial tear following photodynamic \\ therapy (PDT) for subfoveal choroidal \\ neovascularization (CNV) secondary to age- \\ related macular degeneration (AMD). \\ Design Retrospective interventional case \\ series. \\ Methods A retrospective study in an
} institutional practice. We describe seven cases of retinal pigment epithelial (RPE) tear, which developed in seven eyes of seven patients following PDT. All eyes had subfoveal CNV secondary to AMD.

Results Six eyes had occult subfoveal $\mathrm{CNV}$, and one eye had recurrent classic subfoveal CNV. In five patients, the eye that developed the tear was the second eye, whereas the first eye had a disciform scar. In four eyes, the RPE tear developed after one PDT, in one eye the RPE tear developed after the second PDT, and in two eyes the RPE tear developed after the third PDT. In five of seven cases, there was a significant visual deterioration following the RPE tear.

Conclusions RPE tear is a complication that may occur following PDT in particular when the PDT is applied to an occult subfoveal CNV.

Eye (2005) 19, 1315-1324. doi:10.1038/sj.eye.6701765; published online 1 April 2005

Keywords: choroidal neovascularization; retinal pigment epithelial tear; photodynamic therapy
M Goldstein, G Heilweil, A Barak and

A Loewenstein
Introduction

Photodynamic therapy (PDT) is the recommended treatment for patients suffering from subfoveal choroidal neovascularization (CNV) secondary to age-related macular degeneration (AMD). ${ }^{1-4}$ Retinal pigment epithelial (RPE) tear is a known complication of a pigment epithelial detachment (PED), occurring either spontaneously ${ }^{5-9}$ or following laser treatment. ${ }^{10,11}$ Usually there is a significant decrease in vision at the time of tear formation as described previously by Coscas et al. ${ }^{12}$ It was found that the RPE tear usually happens in those cases in which on fluorescein angiogram there is an uneven filling of the PED with a remarkably hypofluorescent paracentral area. Both Coscas and Gass believe that the tear is formed in those cases in which there is a CNV underlying the PED. ${ }^{9,10,12}$ In the ophthalmological literature, we found only five cases reported describing the appearance of RPE tear following PDT for subfoveal CNV secondary to AMD. ${ }^{13,14}$ We wish to describe a series of seven patients who underwent PDT for CNV secondary to AMD and subsequently developed an RPE tear.

\section{Methods}

A retrospective interventional case series $(n=7)$ at a tertiary university retinal clinic was used to describe RPE tear formation following PDT for subfoveal CNV secondary to AMD.

Results

\section{Patient No 1}

An 83-year-old male was referred to our clinic due to decreased vision of 3 weeks duration in
Department of Ophthalmolgy, Tel-Aviv Medical center, Sackler School of medicine, Tel-Aviv, Israel

\section{Correspondance:} M Goldstein, Department of Ophthalmology, Tel-Aviv medical Center, Sackler School of medicine, 6 Weizman street, Tel-Aviv 64239, Israel Tel: + 97236973408 ; Fax: +97236973867 . E-mail: michgold@ netvision.net.il

Received: 3 February 2004 Accepted: 4 October 2004 Published online: 1 April 2005 
the right eye. On examination, visual acuity was 20/200 in the right eye and 20/60 in the left. The anterior segment was normal in both eyes and intraocular pressure was normal. Fundus examination disclosed a normal optic disc with peripapillary atrophy and a large subfoveal PED with suspected CNV on its nasal edge in the right eye, and normal optic disc with peripapillary atrophy, and drusen in the macula in the left eye. On
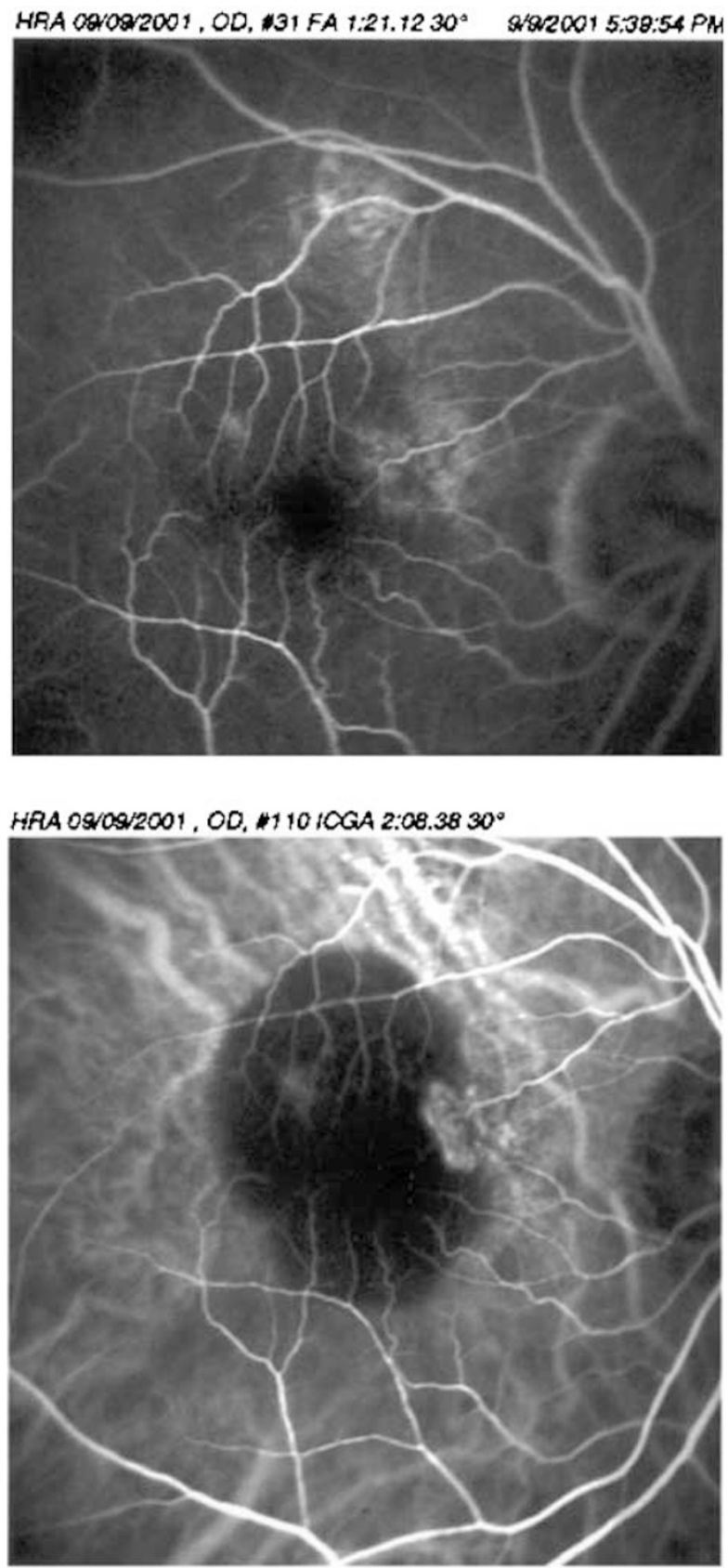

fluorescein angiography (FA), a large subfoveal fibrovascular PED could be detected, and indocyanine green (ICG) angiography demonstrated a large hypofluorescent area consistent with the PED and a bright hyperfluorescent hot spot on the nasal edge of the PED consistent with the CNV (Figure 1). The diagnosis was of occult subfoveal CNV, and PDT was performed. At 6 weeks following the PDT, vision had slightly

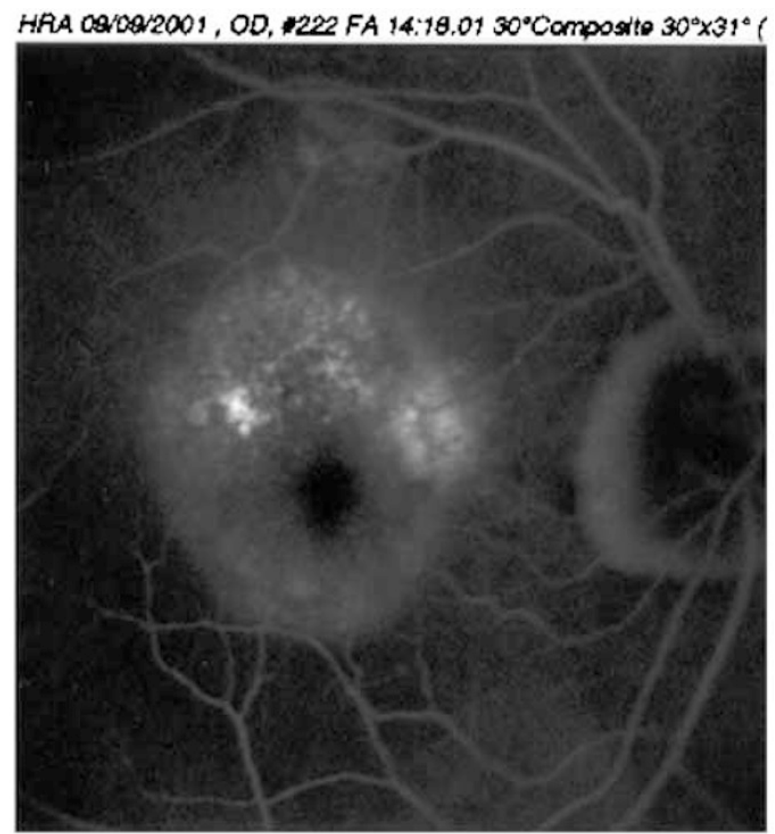

HRA OQOOY2001, OO, \#188 ICGA 17:54.95 30

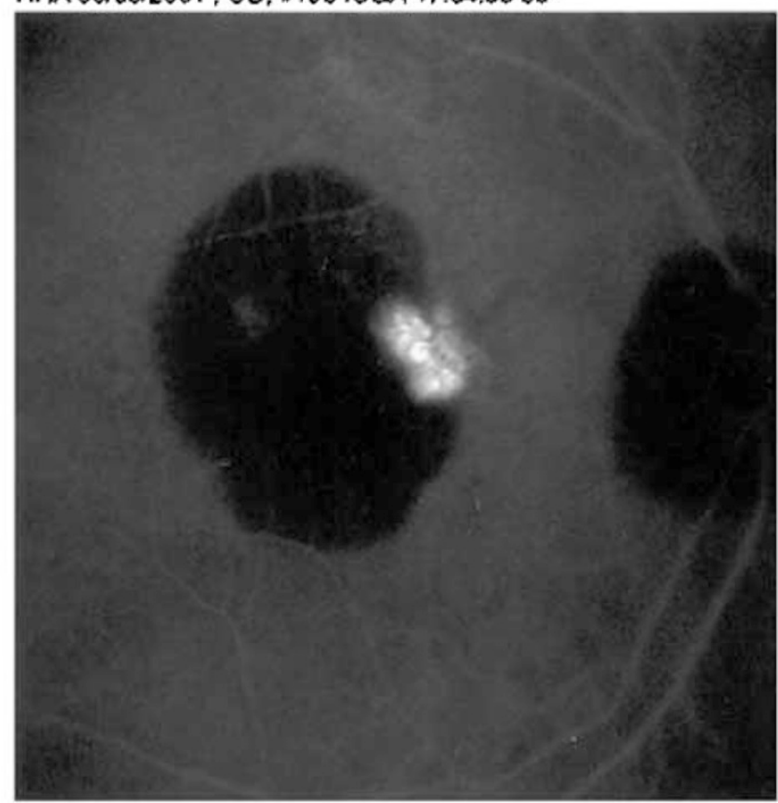

Figure 1 Case 1 - pretreatment of right eye: early-phase FA (top left) shows subfoveal irregular elevation of RPE, with a brighter area of hyperfluorescence nasally. Late-phase FA (top right) shows increased subfoveal hyperfluorescence consisting of fibrovascular PED. Early-phase ICG (bottom left) shows a large hypofluorescent area consisting of PED with a hot spot on its nasal edge. Late-phase ICG (bottom right) shows intense leakage from the CNV located nasally. 

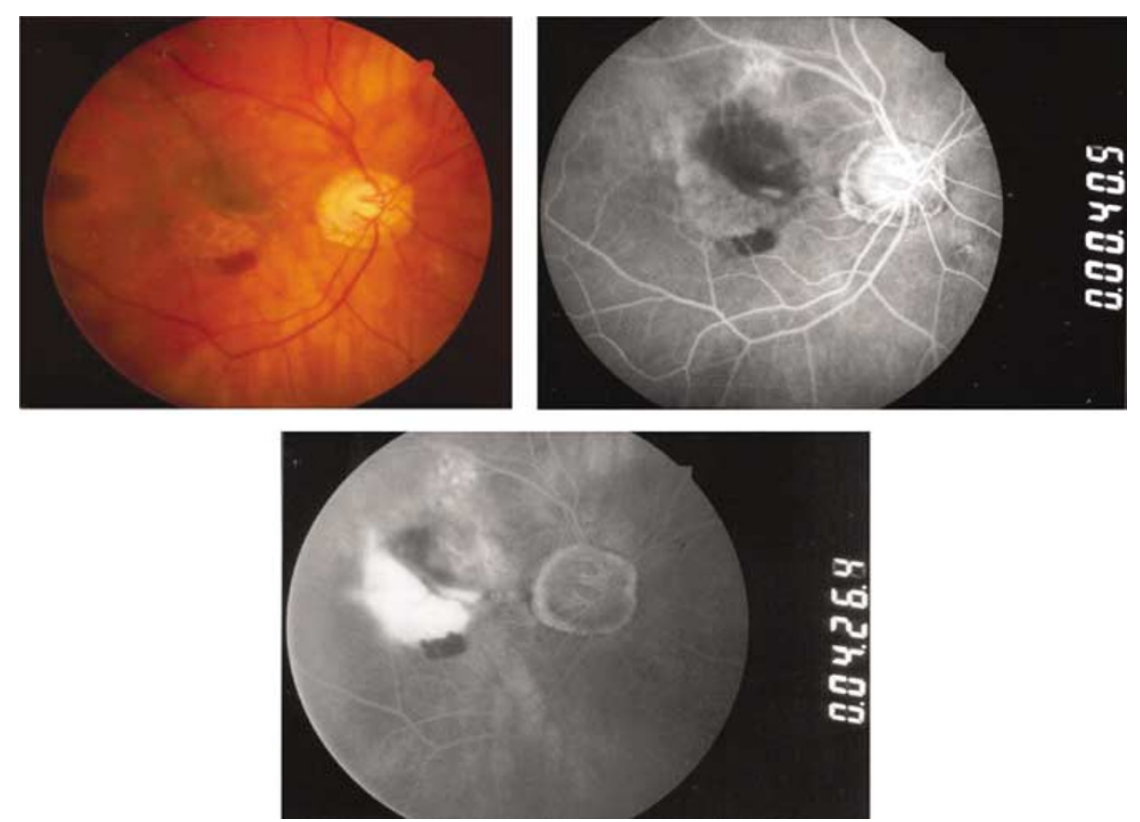

Figure 2 Case $1-6$ weeks after PDT of the right eye: colour fundus photograph (top left) shows the RPE tear with the crescent shape area of absent RPE inferotemporally and the rolled edge of torn pigment epithelium, and some contiguous blood inferior to the tear. Early-phase FA (top right) shows early hyperfluorescence corresponding to the area of RPE loss and hypofluorescence in the area of retracted and organized RPE mound. Late-phase FA (bottom left) shows diffuse hyperfluorescence due to staining.

deteriorated to 20/240 and fundus examination revealed a well-demarcated area inferior to the fovea, which became hyperfluorescent with time lined superiorly by a hypofluorescent border, consistent with the diagnosis of an RPE tear (Figure 2). No further treatment was offered to the patient. At 1 year following the PDT, vision is $20 / 800$ in the right eye and 20/40 in the left, and on fundus examination, a disciform scar in the right and drusen in the left can be seen.

\section{Patient No 2}

A 72-year-old male complained of metamorphopsia of 4 months duration in his left eye. His past ocular history included severe visual loss in his right eye for 7 years due to a disciform scar secondary to AMD. On examination, visual acuity was finger counting in the right eye and $20 / 50$ in the left. The anterior segment was normal in both eyes. Fundus examination disclosed a normal optic disc and a large macular disciform scar in the right eye, and a normal optic disc and a small subfoveal PED with overlying minimal neurosensory detachment in the left. FA of the right eye revealed a disciform scar, and in the left eye, a juxtafoveal fibrovascular PED (Figure 3). The patient underwent PDT with Verteporfin. When he returned for his follow-up visit, 3 months later, vision in the left eye was well preserved at 20/50, but fundus examination disclosed a persistent PED lined by a sharp, well-demarcated area nasally in which the choroidal vessels could be seen diagnosed clinically as RPE tear. FA confirmed the diagnosis of an RPE tear but demonstrated persistent leakage from the subfoveal occult CNV (Figure 4). The patient underwent a second PDT. At 3 months following the second PDT, vision in the left eye remained stable at 20/50, and fundus examination revealed unchanged RPE tear nasal to the fovea, and on FA, persistent leakage from the occult CNV. A third PDT was performed. At 3 months following the third PDT, the patient returned for examination complaining of decreased vision. At that time, vision in the left eye was 20/100. FA revealed an enlarged occult CNV with slightly increased leakage (Figure 5) and a fourth PDT was administered to the patient.

\section{Patient No 3}

A 78-year-old known to suffer from a macular scar in the right eye secondary to AMD, and postlaser treatment for extrafoveal CNV in his left eye 6 months earlier, presented with decreased vision in his left eye. On examination, visual acuity was finger counting in the right eye and 20/200 in the left. Fundus examination disclosed a subfoveal pigment epithelial elevation adjacent to the old laser scar with an overlying neurosensory detachment. FA demonstrated two foci of recurrent subfoveal classic CNV superior and inferior to 

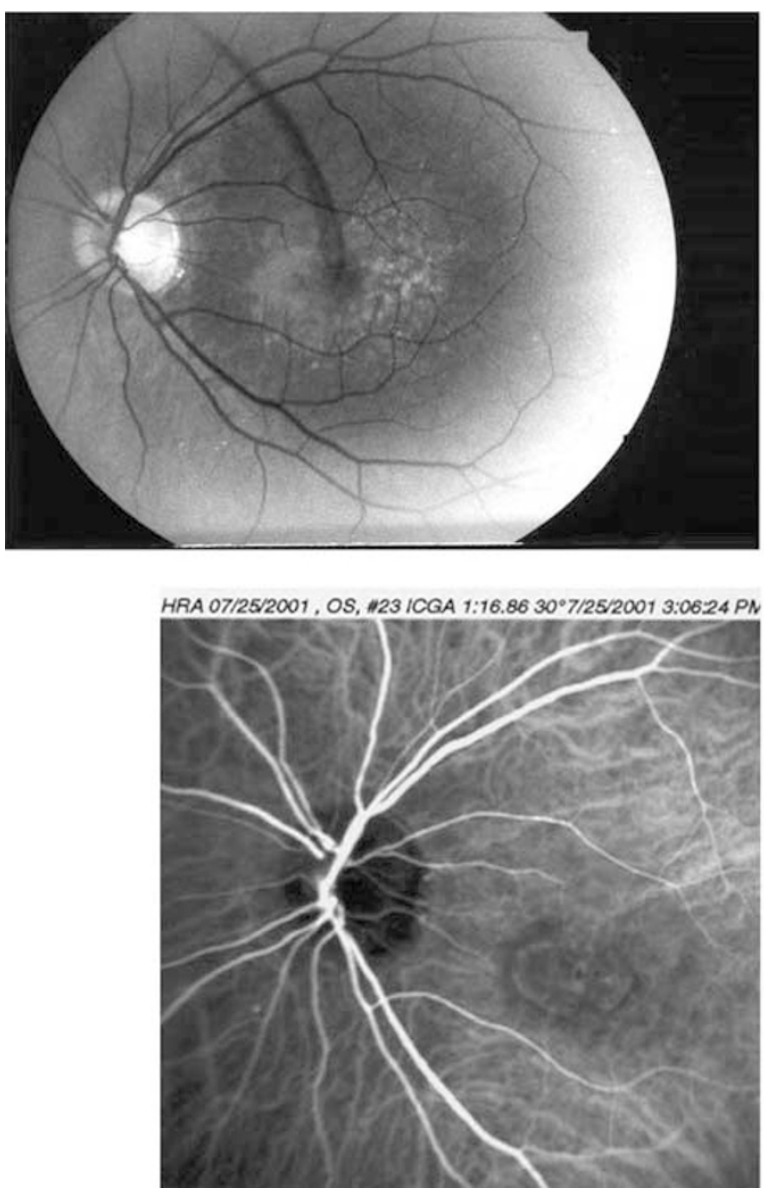
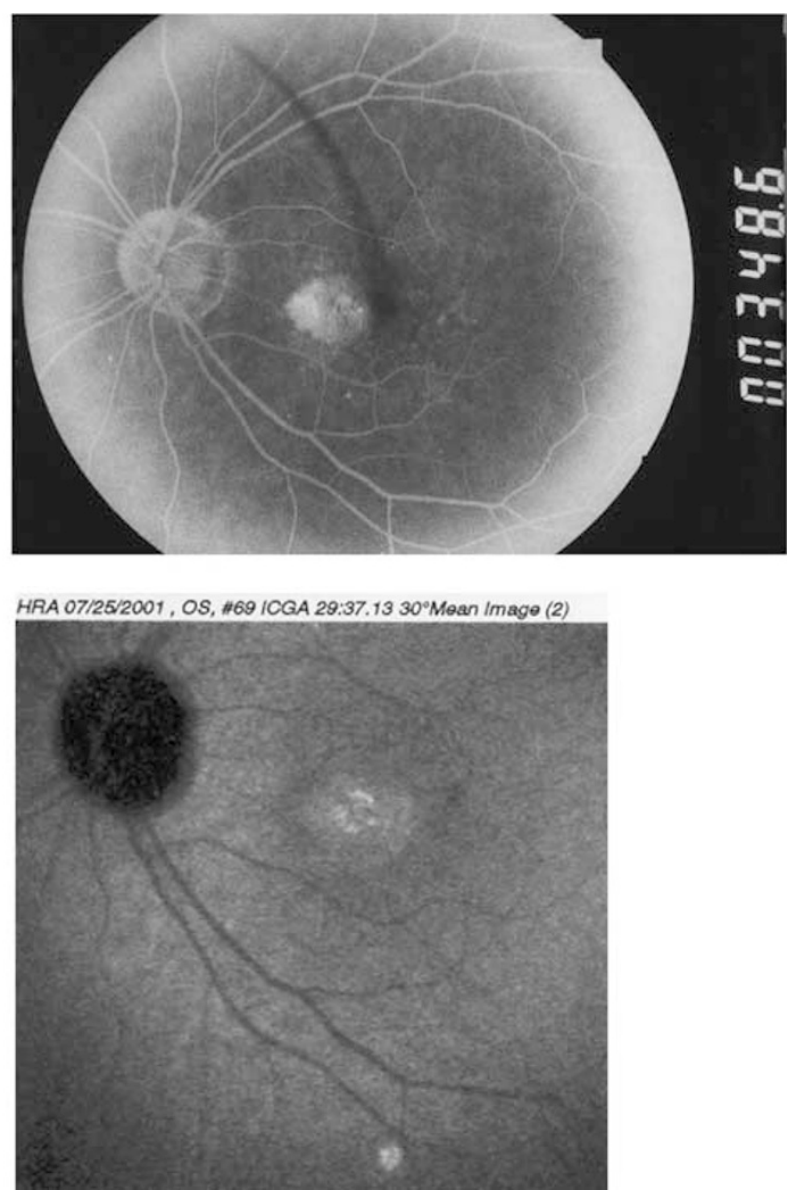

Figure 3 Case 2-pretreatment of left eye. Fundus photograph (top left) shows subfoveal elevation of RPE consisting of CNV and multiple drusen. Mid-phase FA (top right) shows a fibrovascular PED. Early-phase ICG (bottom left) shows a subfoveal hyperfluorescent plaque consisting of occult CNV. Late-phase ICG (bottom right) shows the increased hyperfluorescence due to leakage from $\mathrm{CNV}$.

the old scar with two visible feeder vessels (Figure 6). PDT was applied to the entire lesion followed by focal argon laser treatment directly on the feeder vessel 10 days later. At 3 weeks following the laser treatment, vision decreased to 20/400 and on fundus examination, a well-demarcated area of visible choroidal vessels was seen temporal to the fovea clinically diagnosed as an RPE tear confirmed by FA (Figure 7). No further treatment was offered.

\section{Patient No 4}

A 75-year-old male complained of blurred vision of 3 months duration in his right eye. In his left eye, he was known to suffer from a disciform scar secondary to AMD. On examination, visual acuity was 20/60 in the right eye and light perception in the left. The anterior segment was normal in the right eye, and mature cataract was found in the left eye. Fundus examination disclosed a normal optic disc, subfoveal elevation of the pigment epithelium adjacent to a small subretinal haemorrhage with overlying neurosensory detachment in the right eye. FA of the right eye revealed a juxtafoveal occult CNV

(Figure 8). The patient underwent PDT. On his follow-up visit, vision in the right eye was well preserved at 20/60; nevertheless, fundus examination disclosed an enlarged elevation of the RPE with overlying neurosensory detachment. FA demonstrated an enlarged occult CNV with persistent leakage. The patient underwent a second PDT. At 3 months following the second PDT, vision in his right eye deteriorated to a level of 20/200. Fundus examination revealed a large PED, and on FA persistent leakage from the occult subfoveal CNV (Figure 9).

A third PDT was performed. At 3 months following the third PDT, the patient returned for examination complaining of a further decrease in vision which he had noticed 1 month prior to his visit. At that time vision in the left eye was 20/400, and fundus examination revealed an RPE tear inferior to the fovea confirmed by FA, which demonstrated persistent leakage from the 

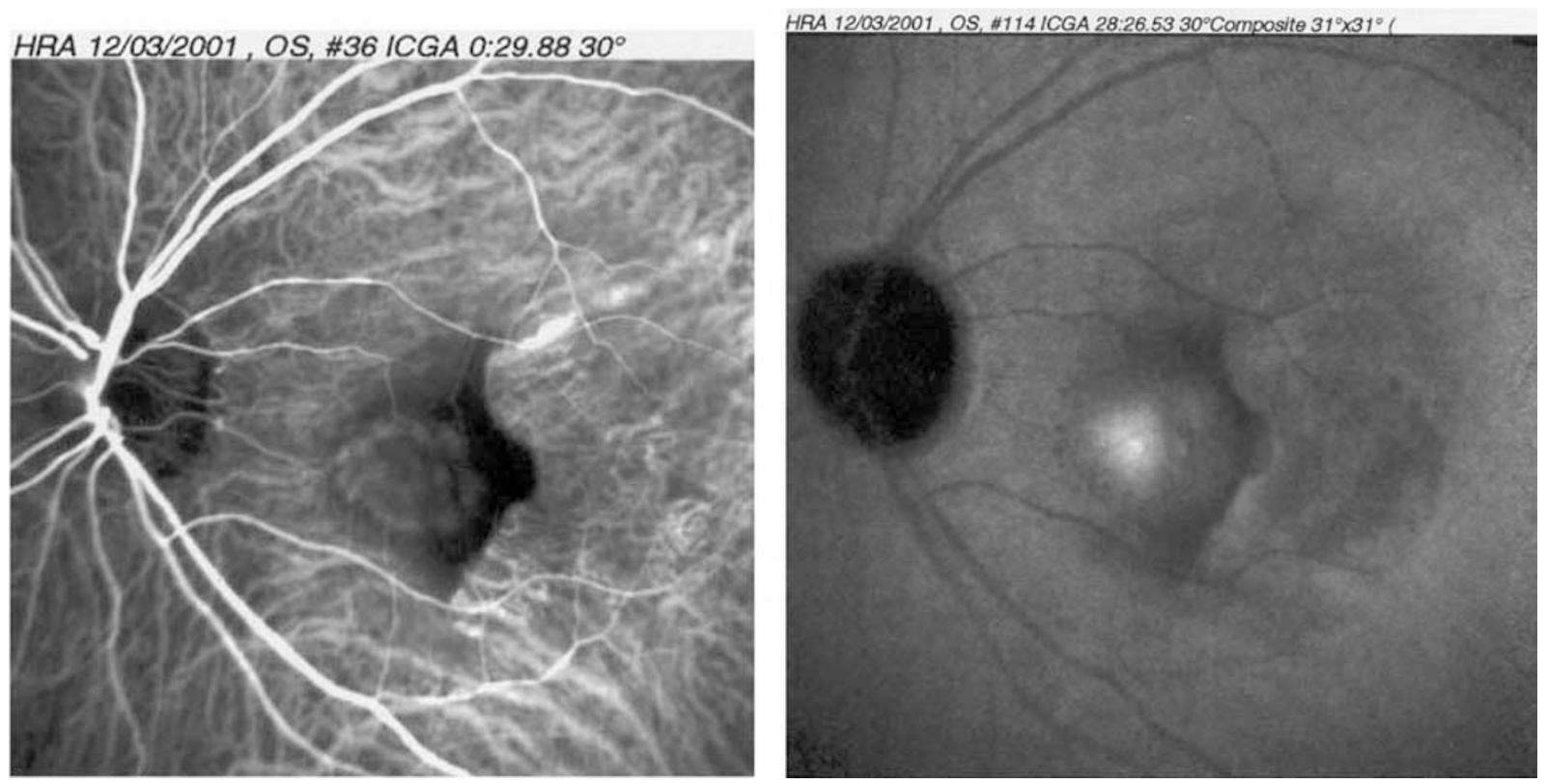

Figure 4 Case 2-3 months after PDT to the left eye. Early-phase ICG (left) shows the sharp demarcation line consisting of the rolled edge of torn RPE and subfoveal hyperfluorescent plaque consisting of persistent occult CNV. Late-phase ICG (right) shows increased hyperfluorescence consisting of leakage from the CNV.
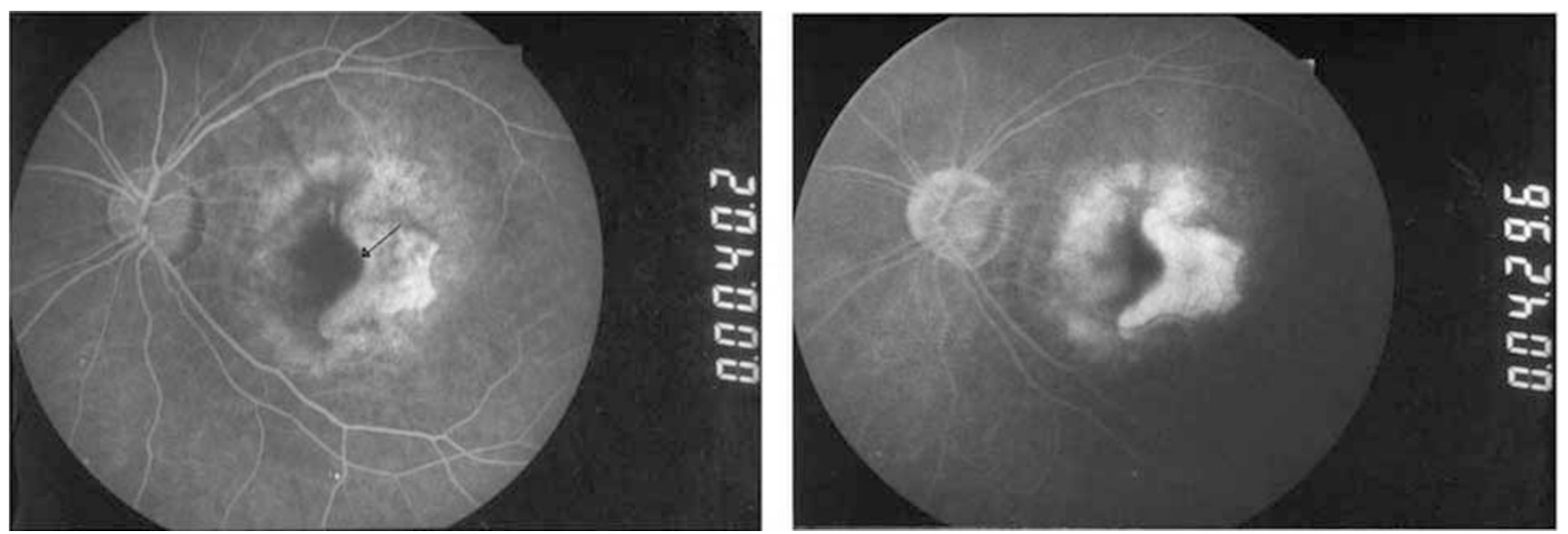

Figure 5 Case 2-3 months after the third PDT to the left eye. Early-phase FA (left) shows the RPE tear (arrow) with hyperfluorescence nasally. Late-phase FA (right) shows diffuse leakage from persistent enlarged occult CNV.

subfoveal CNV (Figure 10). PDT was administered for the fourth time.

\section{Patient No 5}

An 85-year-old male was followed in our retina clinic for intermediate AMD in the right eye and a disciform scar in the left. Visual acuity was 20/50 in the right eye, and finger counting in the left. On his scheduled follow-up visit, he complained of metamorphopsia in the right eye. On examination visual acuity was 20/80. Fundus examination disclosed a subfoveal PED consistent with an occult subfoveal CNV on FA. The patient underwent
PDT. On his 3 months scheduled follow-up visit, visual acuity was 20/100 in the right eye. Fundus examination disclosed an unchanged occult subfoveal CNV confirmed by FA, and the patient underwent a second PDT. At 1 month following the second PDT, the patient complained of a sudden decrease in vision in his right eye. On examination, visual acuity was 20/600 and on fundus examination an RPE tear was seen and confirmed by FA. No further treatment was offered to the patient.

\section{Patient No 6}

An 82-year-old woman complained of decreased vision of 2 months duration in her left eye. In her right eye, she 

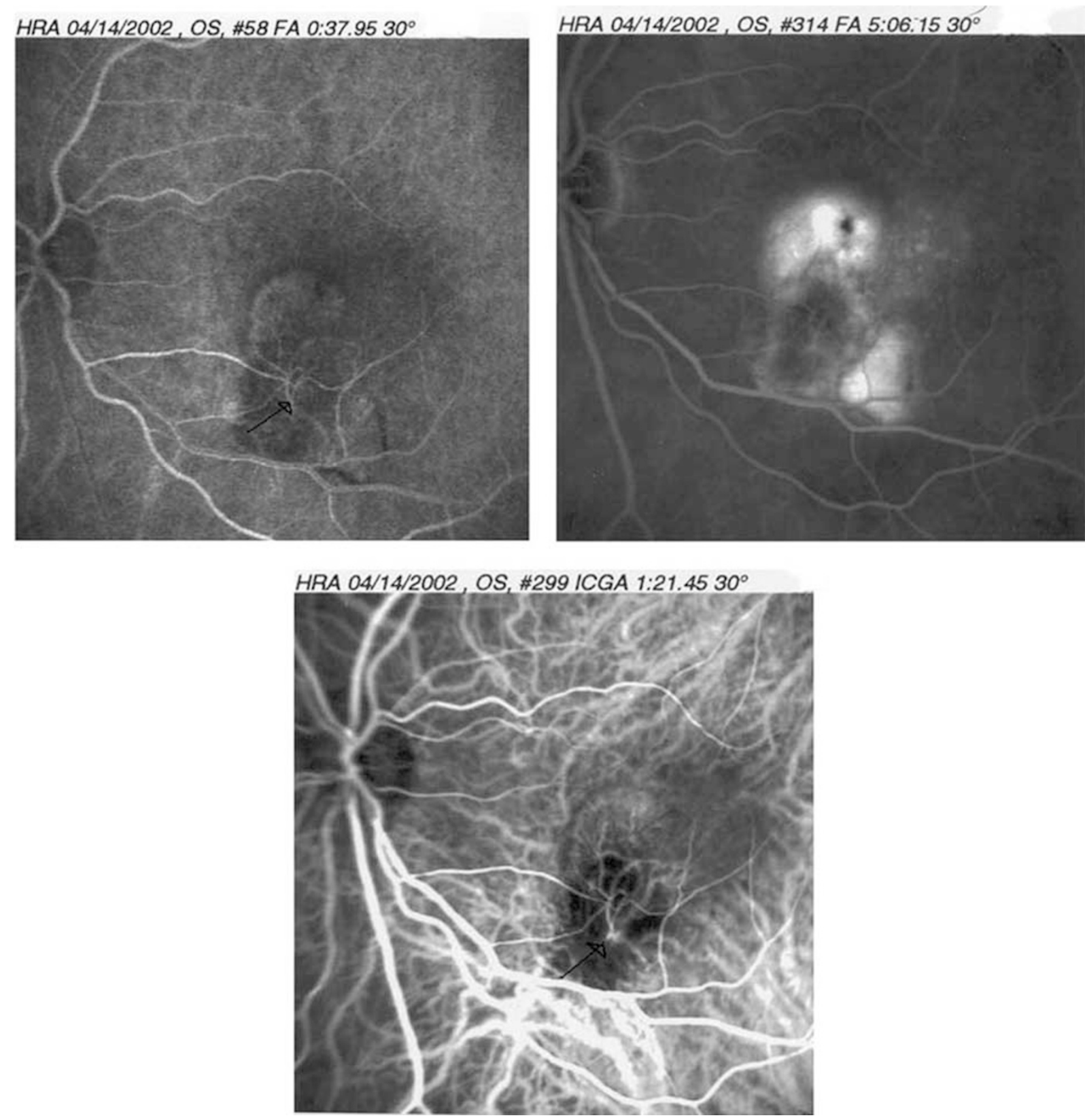

Figure 6 Case 3-6 months after focal argon laser treatment to the CNV of left eye. Early-phase FA (top left) shows recurrent classic subfoveal CNV. A feeder vessel arising from the centre of the laser scar could be identified (arrow). Late-phase FA (top right) demonstrates leakage from the subfoveal CNV. Early-phase ICG (bottom left) demonstrates clearly the feeder vessel (arrow).

suffered from a macular disciform scar secondary to AMD. On examination, visual acuity was finger counting in the right eye and 20/60 in the left. Anterior segment was normal in both eyes except for bilateral nuclear sclerosis of the lens. Fundus examination disclosed a large disciform scar in the right eye, and subfoveal pigment epithelium elevation with overlying minimal neurosensory detachment in the left. FA of the left eye confirmed the diagnosis of occult subfoveal CNV. The patient underwent PDT. Owing to persistent leakage on the FA 3 months post-treatment, a second PDT was performed. Visual acuity 3 months following the second PDT was preserved at 20/80, but due to enlarged CNV and persistent leakage on the FA, she underwent a third PDT. At 6 weeks following her third PDT, she noticed a severe decrease in vision. On examination, visual acuity in the left eye had dropped dramatically to $20 / 400$ and on fundus examination a large RPE tear was observed and confirmed by FA. No further treatment was recommended. 

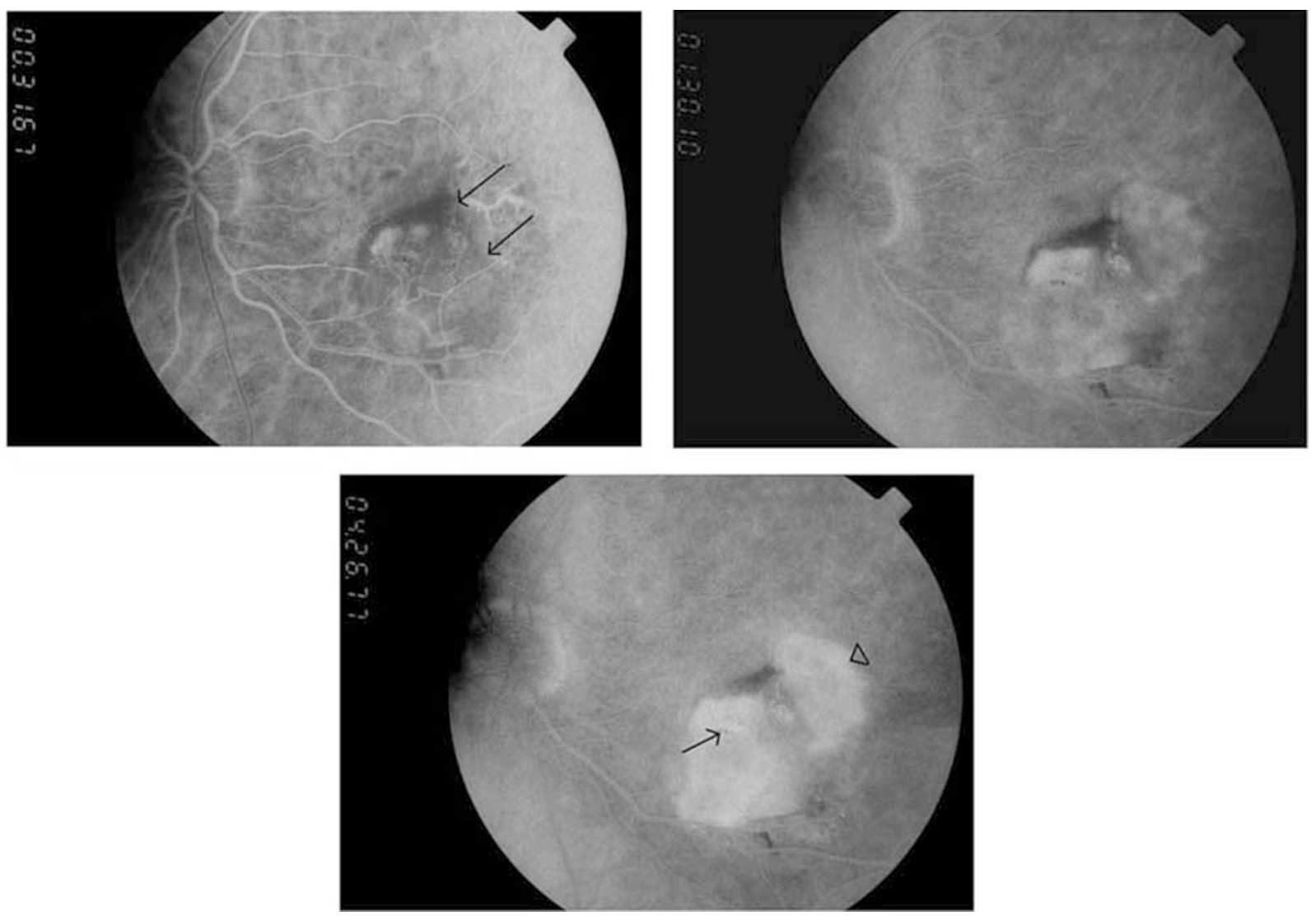

Figure 7 Case 3-4 weeks after PDT and laser treatment of the left eye. Early-phase FA (top left) shows the RPE tear superotemporal to the fovea (arrow) and early subfoveal hyperfluorescence consisting of persistent classic subfoveal CNV. Mid-phase FA (top right) shows increased hyperfluorescence from the CNV. Late-phase FA (bottom left) shows diffuse leakage from the CNV (arrow) and only mild staining superiorly corresponding to the area of RPE loss (arrowhead).
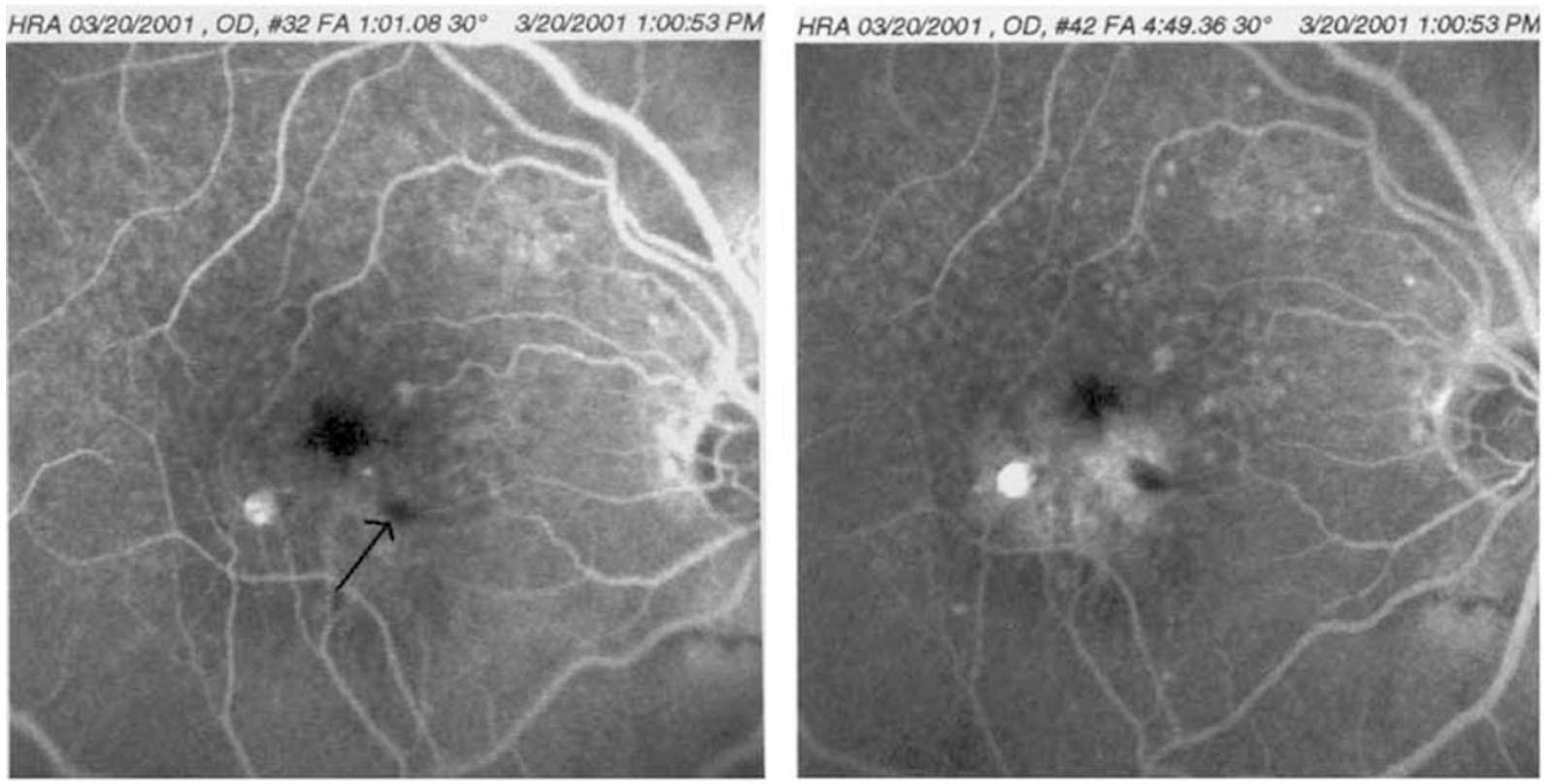

Figure 8 Case 4-pretreatment of right eye. Early-phase FA (left) shows a juxtafoveal fibrovascular PED and a small intraretinal haemorrhage nasally. Late-phase FA (right) shows increased hyperfluorescence due to leakage from the occult CNV. An hyperfluorescent spot with no evidence of leakage can be seen temporally consisting of a small area of geographic atrophy. 

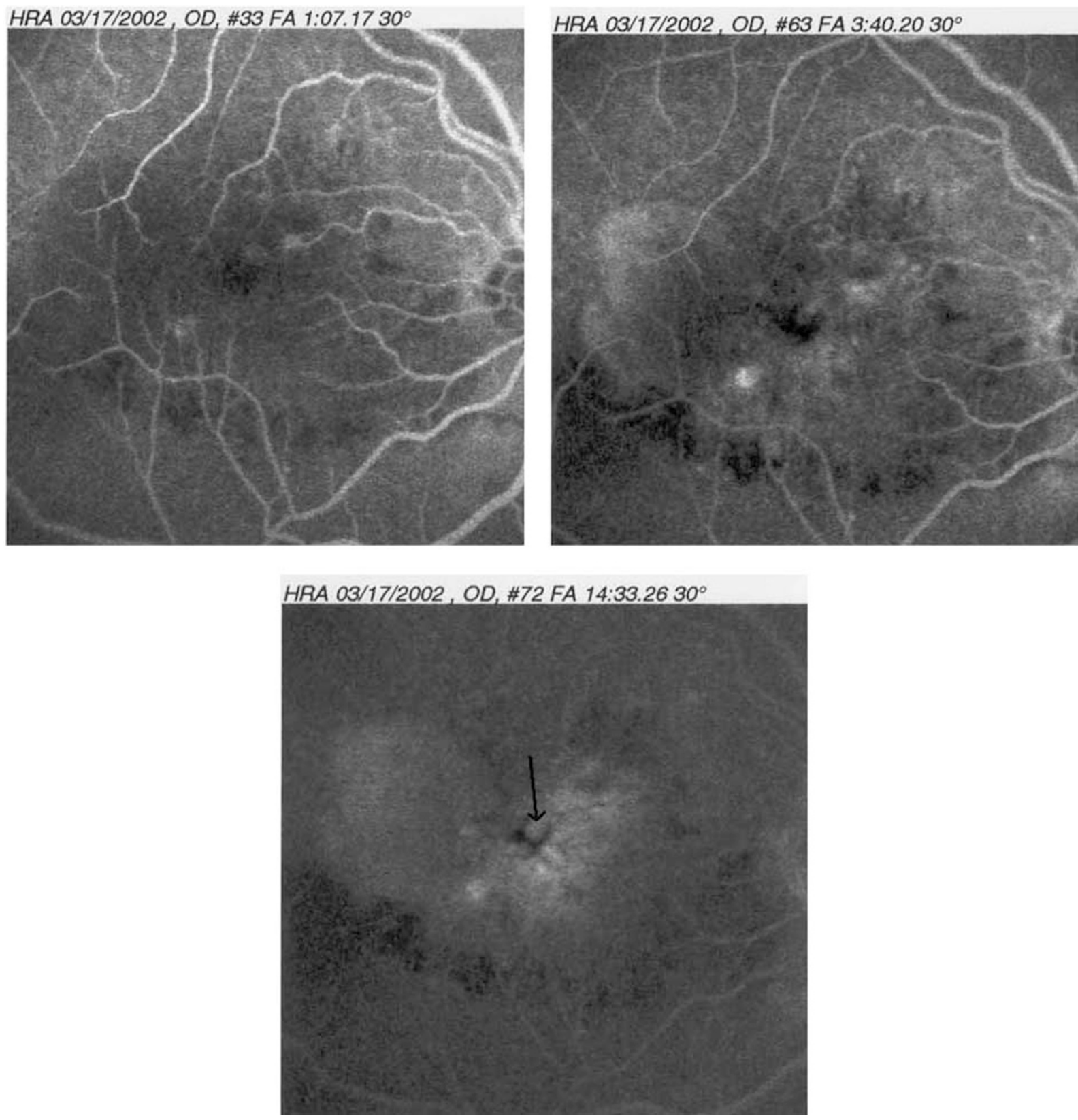

Figure 9 Case 4-3 months after the second PDT to the right eye. Early-phase FA (top left) shows an enlarged fibrovascular PED. Mid-phase FA (top right) shows leakage from the occult CNV with contiguous blood inferiorly. Late-phase FA (bottom left) shows the diffuse leakage from the CNV with secondary CME (arrow).

\section{Patient No 7}

A 73-year-old male complained of mild blurriness of vision in his right eye. On examination visual acuity was $20 / 40$ in the right eye and 20/30 in the left. Anterior segment was normal in both eyes. Fundus examination disclosed subfoveal PED with no evidence of blood or intraretinal lipids in the right eye, and few medium-sized drusen around the fovea were detected in the left. The diagnosis of a subfoveal fibrovascular PED was confirmed by FA. ICG demonstrated a hypofluorescent area consistent with the PED without a hot spot. Since there was no clear evidence of progression, treatment was differed at that stage, the patient was scheduled for follow-up in 3 months time. He returned 6 months later complaining of decreased vision in his right eye. On examination, visual acuity was 20/140 in the right eye. Fundus examination disclosed an enlarged fibrovascular PED with contiguous subretinal haemorrhage. The blood was less than $50 \%$ of the total lesion size, and the patient 


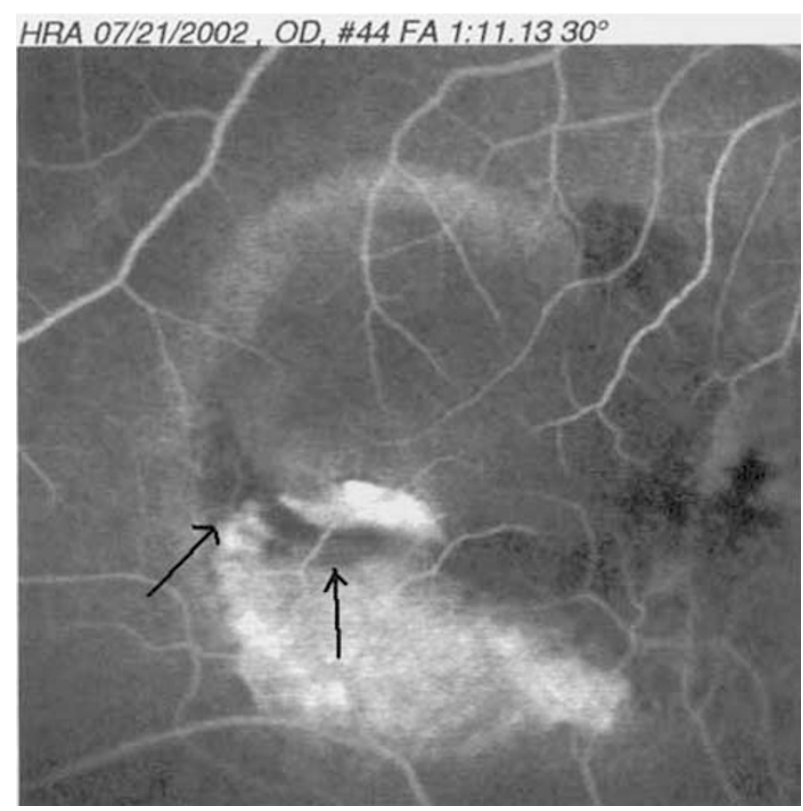

Figure 10 Case $4-3$ months after the third PDT of the right eye. Mid-phase FA shows the RPE tear (arrow).

underwent PDT. On his 10 weeks follow-up visit, visual acuity improved to 20/100 in the right eye, and fundus examination disclosed complete absorption of the subretinal haemorrhage. Nevertheless, an RPE tear was suspected to exist inferior to the fovea. On FA, there was persistent leakage from the subfoveal $\mathrm{CNV}$, and the existence of an RPE tear inferiorly was confirmed. The patient underwent a second PDT with the aiming beam directed to the area of the CNV. At 3 months following the second PDT, visual acuity further improved to 20/80 in the right eye, but there was still subfoveal RPE elevation with overlying neurosensory elevation lined by the RPE tear on the inferior border of the CNV. The patient was offered a third PDT on the CNV.

\section{Discussion}

We report on seven patients with subfoveal CNV secondary to AMD who had developed an RPE tear following PDT. Six of the choroidal neovascular lesions were occult, while one was a recurrent classic CNV. In five of our patients, the eye that had developed the tear was the second eye involved, whereas the first already had a disciform scar with very poor vision.

PDT was recommended in all cases with respect to lesion composition and size, at presentation. ${ }^{1-4}$ All six cases with occult $\mathrm{CNV}$ had shown evidence of progression such as visual deterioration and/or intraretinal blood and/or increase in lesion size and therefore were eligible for treatment according to the VIP results. ${ }^{3}$ One patient had a vision of 20/50 and a CNV smaller than 4 disc area and all other six patients had pretreatment visual acuity of 20/60-20/200. According to the VIP results, ${ }^{3}$ in those selected cases with visual acuity $\leqslant 20 / 50$ and/or lesion size smaller than 4 DA, there is significant treatment benefit in maintaining stable vision ( $51 \%$ in treated vs $25 \%$ in controls). In four cases (cases 1-3, 7) the RPE tear developed following the first PDT, in one case (case 5) following the second PDT, and in two cases (cases 4,6) following the third PDT.

It has been previously described that retinal pigment epithelial tear can occur spontaneously or following laser treatment..$^{5-12}$ Few small case series have described the occurrence of retinal pigment epithelial tear after PDT. ${ }^{13-15}$ Gelisken et $a l^{13}$ reported the first case of an RPE tear 1 month following PDT for classic subfoveal CNV. Pece et $a l^{14}$ reported four cases of RPE tear developing following PDT for occult CNV with PED. In all of their cases, the RPE tear developed within 7 days after PDT, two cases had decreased vision to a level of 20/200, one case had stable vision and one case had improved vision in spite of the development of an RPE tear. Srivastava and Sternberg ${ }^{15}$ reported on one case of an RPE tear in a young patient who underwent PDT for juxtafoveal CNV secondary to high myopia. The tear had occurred 6 weeks following her second PDT with a severe decrease in vision to $20 / 400$. In our series, five patients suffered severe visual loss to 20/400-20/800 following the development of the tear, one patient maintained a vision of 20/50, and one patient had improved vision from 20/ 140 to 20/80 despite the development of an RPE tear. The time interval between PDT and the tear ranged from 3 weeks following the first PDT to 8 weeks following the third PDT.

Hoskins et $a l^{6}$ suggested that the RPE detaches from its underlying basement membrane at the area of highest elevation. Gass ${ }^{9}$ hypothesizes that the mechanism involves first CNV, which gradually elevates the basement membrane together with the RPE. The leaking $\mathrm{CNV}$ causes a serous detachment of the adjacent RPE which by the growing pressure of the serous fluid beneath the RPE detaches the RPE away from the opposite side of the neovascular complex where the RPE is firmly attached. The pathogenesis of tear formation post-thermal laser as proposed by Gass ${ }^{10}$ is a sudden contraction of the CNV secondary to the heat generated by the laser. Kramer et $a l^{16}$ demonstrated RPE damage following PDT in monkeys. The damage to the RPE is probably caused by a direct photochemical effect due to early accumulation of Verteporfin in those cells. ${ }^{17,18}$ The damaged RPE cells usually showed recovery within 3-4 weeks but with some loss of pigmentation and rearrangement in multiple layers. ${ }^{19}$ Repeated treatments using the 6-mg $/ \mathrm{m}^{2}$ Verteporfin dose in monkeys showed recovery of choriocapillaris, with mild retinal pigment 
epithelium and outer photoreceptor damage at 6 weeks. ${ }^{20}$ In all cases described by Pece et al, ${ }^{14}$ the tear occurred within 1 week of treatment and in three of our cases, the tear occurred within 3-6 weeks post-treatment, and in the four other cases, the tear was seen on the follow-up visit so the exact time could not be appreciated. As described previously, in those few weeks following PDT, the RPE cells are vulnerable and may rupture. SchmidtErfurth $e a^{21}$ found that the choroid exposed to photoactivation exhibited choriocapillary occlusion; however, progressive recanalization was documented within 4-12 weeks after both single and multiple PDT. Michels and Schmidt-Erfurth ${ }^{22}$ reported recently on the vascular changes in CNV immediately after PDT evaluated by confocal FA and ICG angiography. They observed no immediate occlusion of the CNV. At 1 day after PDT, the CNV even though reaching its minimum size, was accompanied by a massive exudation originating from the $\mathrm{CNV}$ and surrounding choroids, which slowly resolved by 1 week. These findings may explain the formation of RPE tear following PDT. It may be that the early increased exudation beneath the RPE on the one hand and the altered choroidal perfusion on the other hand may weaken the adhesion of the RPE and increase traction on one edge of the RPE elevation causing the tear formation.

RPE tear formation is not a common complication following PDT. Our impression is that the risk for development of the RPE tear may be higher when the PDT is applied to occult CNV or PED, rather than to classic $\mathrm{CNV}$, and more common in fellow eyes of patients who suffer from a disciform scar in their first eye.

\section{References}

1 Treatment of Age-Related Macular Degeneration with Photodynamic Therapy (TAP) Study Group. Photodynamic therapy of subfoveal choroidal neovascularization in age related macular degeneration with verteporfin; one year results of two randomized clinical trials-TAP report 1. Arch Ophthalmol 1999; 117: 1329-1345.

2 Treatment of Age-Related Macular Degeneration with Photodynamic Therapy (TAP) Study Group. Photodynamic therapy of subfoveal choroidal neovascularization in age related macular degeneration with verteporfin; two year results of two randomized clinical trials-TAP report 2 . Arch Ophthalmol 2001; 19: 198-207.

3 Verteporfin in Photodynamic Therapy (VIP) Study Group. Photodynamic therapy of subfoveal choroidal neovascularization in age-related macular degeneration with verteporfin: two year results of a randomized clinical trial-VIP report 2. Am J Ophthalmol 2001; 131: 541-560.

4 Treatment of Age-Related Macular Degeneration with Photodynamic Therapy (TAP) Study Group. Verteporfin therapy for subfoveal choroidal neovascularization in agerealted macular degeneration. Three-year results of an open-label extension of 2 randomized clinical trials - TAP Report 5. Arch Ophthalmol 2002; 120: 1307-1314.

5 Meredith TA, Braley RE, Aaberg TM. Natural history of serous detachments of the retinal pigment epithelium. Am J Ophthalmol 1979; 88: 643-651.

6 Hoskins A, Bird AC, Sehmi K. Tears of detached retinal pigment epithelium. Br J Ophthalmol 1981; 65: 417-422.

7 Poliner LS, Olk RJ, Burgess D, Gordon ME. Natural history of retinal pigment epithelial detachments in age-related macular degeneration. Ophthalmology 1986; 93: 543-550.

8 Elman MJ, Fine SL, Murphy RP, Patz A, Auer C. The natural history of serous retinal pigment epithelial detachment in patients with age-related macular degeneration. Ophthalmology 1986; 93: 224-230.

9 Gass JDM. Pathogenesis of tears of the retinal pigment epithelium. Br J Ophthalmol 1984; 68: 513-519.

10 Gass JDM. Retinal pigment epithelial rip during krypton red laser photocoagulation. Am J Ophthalmol 1984; 98: 700-706.

11 Yeo JH, Marcus S, Murphy RP. Retinal pigment epithelial tears. Patterns and prognosis. Ophthalmology 1988; 95: 8-13.

12 Coscas G, Koenig F, Soubrane G. The pretear characteristics of pigment epithelial detachments. A study of 40 eyes. Arch Ophthalmol 1990; 108: 1687-1693.

13 Gelisken F, Indhoffen W, Partsch M, Schneider U, Kreissing I. Retinal pigment epithelial tear after photodynamic therapy for choroidal neovascularization. Am J ophthalmol 2001; 131: 518-520.

14 Pece A, Introini U, Bottoni F, Brancato R. Acute retinal pigment epithelial tear after photodynamic therapy. Retina 2001; 21: 661-665.

15 Srivastava SK, Sternberg Jr P. Retinal pigment epithelial tear weeks following photodynamic therapy with verteporfin for choroidal neovascularization secondary to pathologic myopia. Retina 2002; 22: 669-671.

16 Kramer M, Miller JW, Michaud N, Moulton RS, Hasan T, Flotte TJ et al. Liposomal benzoporphyrin derivate verteporfin photodynamic therapy. Selective treatment of choroidal neovascularization in monkeys. Ophthalmology 1996; 103: 427-438.

17 Schmidt-Erfurth U, Hasan T. Mechanisms of action of photodynamic therapy with verteporfin for the treatment of age-related macular degeneration. Surv Ophthalmol 2000; 45: 195-214.

18 Haimovici R, Kramer M, Miller JW, Hasan T, Flotte TJ, Gragoudas ES et al. Localization of lipoprotein-delivered benzoporphyrin derivate in the rabbit eye. Curr Eye Res 1997; 16: 83-90.

19 Husain D, Kramer M, Kenny AG, Michaud N, Flotte TJ, Gragoudas ES et al. Effects of photodynamic therapy using verteporphin on experimental choroidal neovascularization and normal retina and choroid up to 7 weeks after treatment. Invest Ophthalmol Vis Sci 1999; 40: 2322-2331.

20 Reinke MH, Canakis C, Husain D, Michaud N, Flotte TJ, Gragoudas ES et al. Verteporphin photodynamic therapy retreatment of normal retina and choroid in the cynomolgus monkey. Ophthalmology 1999; 106: 1915-1923.

21 Schmidt-Erfurth U, Michels S, Barbazetto I, Laqua H. Photodynamic effects on choroidal neovascularization and physiological choroids. Invest Ophthalmol Vis Sci 2002; 43: 830-841.

22 Michels S, Schmidt-Erfurth U. Sequence of early vascular events after photodynamic therapy. Invest Ophthalmol Vis Sci 2003; 44: 2147-2154. 